\title{
Measurement of Deformation for Carbon Fiber Composite during Tensile Load Using Digital Image Correlation Method
}

\author{
Chien-Ching Ma ${ }^{1}$, Ching-Yuan Chang ${ }^{2}$ \\ ${ }^{1}$ Department of Mechanical Engineering, National Taiwan University \\ Taipei 106, Taiwan, Republic of China \\ ccma@ntu.edu.tw; chang@mail.ntut.edu.tw \\ ${ }^{2}$ Department of Mechanical Engineering, National Taipei University of Technology \\ Taipei 106, Taiwan, Republic of China
}

\section{Extended Abstract}

Carbon fiber reinforced polymer is one of burgeoning artificial material used in aerospace and industry because of its outstanding performance of high strength and light weight. Unlike ductile materials, which display distinct necking before breaking, carbon fiber composite materials are brittle and break without significant necking when they reach their tensile strength. They show almost no plastic deformation prior to breaking, and their tensile strength is limited by defects, such as fiber breakage and interfacial delamination. Based on experimental observation, the inhomogeneity within individual carbon fiber fluctuate the mechanical performance when the composited material undergoing tensile load. The unpredictable and unexpected rapture of carbon fiber directly reduces the tensile strength of the commercial specimen for practical usage. Material testing system with strain gauge and extensometers provides stress-strain relation, but is unable to present full-field distribution of displacement and is also unable to provide information during the interior rapture arising. Thus, this study uses high-resolution imaging system to record complete information during the composite plate under tensile test and then uses digital image correlation method to decrypt displacement and strain for both horizontal and vertical directions. The quantitative analysis based on image processing method not only demonstrates the noncontact superiority of visualizing deformation field during partial rapture of fiber occurring, but also gives accurate value of Poisson's ratio during tensile load. In-situ measurement of the proposed method provides valuable data for both academic research and industrial application, and yields biaxial strain both in macroscopic and microscopic scale for investigating mechanical strength and tensile ductility of commercial composited plate.

Digital image correlation (DIC) is one of optical metrology based on experimental mechanics and image processing technology, and its video-based nature enables full-field measurements for the academic research of material science and mechanical engineering. The non-contact feature facilitates the preparation of specimen for measuring engineering strain on the deformed surface. The DIC metrology is valid in the environment of nature light and is suitable for the in-situ measurement of material testing. Compared with conventional contact sensors, the DIC method is able to provide raw data of biaxial displacement of arbitrarily selected points, and retrieves horizontal strain and vertical strain following numerical difference.

This study uses the self-developed DIC program to decrypt (a) biaxial displacement, (b) biaxial strain (c) Poisson's ratio (d) disturbance by micro breaking and (e) full-field displacement of a deformed CFRP specimen. The proposed DIC program is based on matrix calculation and provides both high accuracy and high efficiency for embodying zero mean normalized cross correlation (ZNCC) criterion. In the DIC technique, images of the specimen during the tensile test were captured using a digital camera at a sampling frequency of $86 \mathrm{fps}$. We then conducted measurements and analysis using the strain gauge. The consistent results obtained from DIC method, MTS system and strain gauge have demonstrated that the proposed matrixbased DIC criterion is able to measure displacement and strain precisely. Simultaneous measurement of biaxial displacement yields quantitative value for investigating the microscopic deformation in a local region in comparison of macroscopic inspection obtained from MTS system. The stress-strain curve and Poisson's ration gives roadmap for improving mechanical design refining chemical formula of commercial CFRP specimen. 